\title{
USES AND APPLICATIONS OF UBUNTU: A TECHNICAL GUIDE
}

\author{
Ilias Gkrekos \\ Dept. of Industrial Design \\ and Production \\ Engineering, \\ University of West Attica \\ Athens, Greece
}

\author{
Avraam Chatzopoulos \\ Dept. of Industrial Design \\ and Production \\ Engineering, \\ University of West Attica \\ Athens, Greece
}

\author{
Michail Papoutsidakis \\ Dept. of Industrial Design \\ and Production \\ Engineering, \\ University of West Attica \\ Athens, Greece
}

\author{
Wai Kong Lee \\ UTAR University \\ Dept. of Information and \\ Communication Technology \\ Kampar, Malaysia
}

\begin{abstract}
This paper deals with the uses and applications of the Ubuntu operating system. This work is divided into two parts. The first part is about introducing which will be presented what is the computing system, operating system, kernel, UNIX, GNU/Linux, the advantages and disadvantages of GNU/Linux, the shell, the terminal, distribution, GNU/Linux desktop environment and the GNU/Linux file system and some introductory things about the Ubuntu operating system. The second part concerns only the Ubuntu operating system for the Ubuntu Desktop version. In particular, you first learn how to download and install it. The following are the desktop, its programs and applications and network connections. Also refer to hardware and software. In addition, advanced Ubuntu functions are recorded and possible problems are addressed. Finally, make the presentation of Ubuntu derivatives.
\end{abstract}

\section{Keywords - UBUNTU, Operating system, GNU/Linux}

\section{INTRODUCTION}

\section{A. Computing system -}

The computer system consists of two parts: hardware and software. Hardware is defined as the set of devices included in the computing system. The software refers to all the programs that the computing system can perform. The software is divided into two pieces. The first part refers to the operating system and the second part refers to the applications (programs) [1].

\section{$B$. Computing system -}

The operating system is a set of programs that accepts the commands given by the user and translates these commands into the necessary processes they need to execute [1].

\section{Kernel -}

When referring to the kernel we actually refer to the basic part of an operating system. This section interferes with the hardware of the computer. The kernel is the one that manages its system resources (CPU, Memory, Devices) which is automatically done, allocates them to the executable applications and ensures that they run smoothly. Finally, he is responsible for the communication between software and hardware. Kernels are divided into monolithic kernels, microkernels, hybrid kernels and exokernels [2].

\section{UNIX -}

UNIX is defined as a multiuser and multitasking operating system. The development of UNIX was made by AT \& T Bell's laboratories in the 1960s and 1970s. The UNIX operating system is composed of three pieces. The first piece refers to the kernel. The second part refers to the shell that acts as the intermediate link between the user and the kernel. Finally, the third part refers to the rest of the programs. The features of UNIX are multiuser, multitasking and timesharing [3].

\section{E. GNU/Linux -}

The GNU project began with the aim of developing an operating system that will be free software. "Free software means that users have the freedom to perform, copy, distribute, study, change and improve the software". This work was begun in September 1983 by Richard Stallman and his development began in January 1984.More specifically, GNU is an operating system that is similar to a UNIX operating system but unlike UNIX, GNU is free software. The GNU name you are going to "from the backward acronym" GNU's Not Unix". Because as we mentioned GNU is a functional system similar to the UNIX operating system its system design is modular. This design makes it possible to import software components from other manufacturers into the GNU.GNU systems nowadays use the Linux kernel and this combination gives us the GNU/Linux operating system. GNU/Linux is an operating system made up of free software. It is similar to the UNIX operating system, except that the entire source code is written as free software under the GNU General Public License. Simply put, GNU/Linux is an open source operating system. GNU/Linux gives the user a variety 
of distributions that vary from one another. The GNU General Public License will often see it referenced GNU GPL or GPL is a free software license. This license was created by Richard Stallman for the GNU project. This license was essentially implemented for the registration of GNU project programs. The latest version of the license is version 3 (GPLv3) [4], [5].

\section{F. Advantages-Disadvantages of GNU/Linux -}

The advantages are [6], [7]:

- It is a free and open source operating system.

- It is portable on any hardware platform.

- Multitasking and multiuser.

- It has strong support for network functionality.

- It has a wide variety of distributions where each user makes his choice according to his needs.

- It has very good community support in case of troubleshooting you encounter and ask for their solution.

- Can be used on old computers and make them respond satisfactorily so they are useful again.

- It is safe, very stable and versatile and less vulnerable to malware.

- It has less chance of collapsing than Windows because it is difficult to attack viruses, spyware, trojans, etc., which have the effect of greatly reducing the performance of a computer and because GNU / Linux does not have a registry like Windows it can't handle such errors that have the effect of slowing down the computer over time.

The disadvantages are [6], [7]:

- It's not very user-friendly if it does not have the good knowledge of these kinds of operating systems and especially for beginners with computer literacy.

- Many Windows operating system programs do not run on the GNU/Linux operating system, and only run them through compatibility programs that allow them to run (for example, Wine).

- Limited range in games.

\section{G. Shell -}

The shell is located between the kernel and the user. It reads the commands given by the user which are entered directly by the user via a keyboard or they are read by a file called a shell script or shell program. The shell reads the commands per line through the script line and then searches these commands inside the system. The main purpose but the shell is providing a user interface in addition to transfer orders to the core. It is also a programming language with its own commands and has the ability to receive variables. Summing up the shell we could classify it as a program that receives commands and instructs the operating system to execute these commands. The shell types in a UNIX system are $s h, b a s h, c s h, t c s h$ and $k s h$ [8].

\section{H. Terminal -}

Operating systems have two types of user interface. The first type is the graphical user interface (GUI). The graphical user interface (GUI) refers to the desktop, menu, windows, and toolbars where browsing and tasks are done using your mouse. The second type and old type is the command line interface (CLI). The terminal is the CLI in an operating system. Through the terminal we can perform tasks on an operating system and control it only with commands that we type from our keyboard. Through the terminal we have access to the shell.

When we open the terminal window and type a command, the shell reads this command and shows us the result [9].

\section{Distribution -}

Distribution is the kernel of Linux and a collection of software and together they form an operating system. Each distribution uses the kernel for kernel but the software you install by default in each distribution varies according to the goals and the areas it wants to focus on. This results in several distributions that vary from one another. There are distributions that are supported by volunteer communities, and this has the effect of distributing them free of charge, and there are specific distributions supported by companies where a fee for subscription and support is required. There are distributions that are designed to work on desktops and laptops, and there are distributions that are designed to run on servers [10].

\section{J. The GNU/Linux Desktop Environment -}

The most common desktop environments we encounter in a distribution of a GNU/Linux operating system: GNOME, Unity, KDE, LXDE, Budge, MATE, Xfce, Cinnamon, LXQT and Pantheon [11].

\section{$K$. File System GNU/Linux -}

The GNU/Linux directory structure could be categorized as a tree. Each directory is also called a folder and may include other directories. Directories in GNU/Linux are separated from each other by a forward vertical line /. The main folders on a GNU/Linux system are the following [12]:

- I: Each directory and file in our system is located under the / directory. From this list starts the GNU/Linux file system hierarchy and we could classify this directory as the root directory or root of the file system

- /bin: In this folder there are binary files and other executable programs. They are common programs that are managed by the system, system administrator, and users

- letc: This folder contains the configuration files that control how the operating system or applications work.

- /home: Every user on a GNU/Linux system has a subdirectory that refers to his account (user account). 


\section{International Journal of Engineering Applied Sciences and Technology, 2019 \\ Vol. 4, Issue 5, ISSN No. 2455-2143, Pages 444-454 \\ Published Online September 2019 in IJEAST (http://www.ijeast.com)}

This is this folder. For example, we have set ilias user account so this directory is displayed as follows: /home/ilias.

- /opt: This folder contains the optional software or third-party software.

- /tmp: This folder is used for temporary space by the system.

- /usr: This folder contains the user's programs and read-only data.

- /var: This folder stores all variables and temporary files created by users, such as log files.

\section{The Ubuntu operating system -}

Ubuntu is a GNU/Linux-based operating system. It's actually a GNU/Linux distribution. Ubuntu is an open source and free operating system and is provided for free. The creation of Ubuntu began in 2004 based on the Debian distribution architecture. Ubuntu is sponsored by Canonical LTD, created by Mark Shuttleworth a businessman from South Africa. It works with the GNU General Public License (GPL). Because of this, all of its installed application software is free software. Ubuntu is released in two versions where one version refers to the most recent standard version of the operating system and the other one refers to the most recent version of long term support (LTS). Ubuntu is available for desktops and laptops via Ubuntu Desktop. It is also available for servers via Ubuntu Server. It works in Intel, AMD, and ARM architectures. Uses for APT updates and package management dpkg, APT and snappy. Its kernel is monolithic since it is a GNU/Linux system. It is available in over 55 languages and its official website is https://www.ubuntu.com/ [13].

\section{DOWNLOAD AND INSTALL UBUNTU}

\section{A. Getting Ubuntu -}

Latest version of Ubuntu's operating system is 16.04 LTS and it can be downloaded from https://www.ubuntu.com/download [14].

\section{B. Minimum system requirements -}

Ubuntu's minimum system requirements are the following [14]:

- $1 \mathrm{GHz}$ x86 processor (Pentium 4 or better).

- $1 \mathrm{~GB}$ of system memory (ram).

- $\quad 8.6 \mathrm{~GB}$ of disk space (at least $15 \mathrm{~GB}$ is recommended).

- Video support capable of $1024 \times 768$ resolution.

- Audio support (recommended, but not required).

- An Internet connection (highly recommended, but not required).

\section{Burn ISO Ubuntu -}

Figure 1 shows the process of burning Ubuntu's ISO image to a DVD or bootable USB drive using PowerISO software.
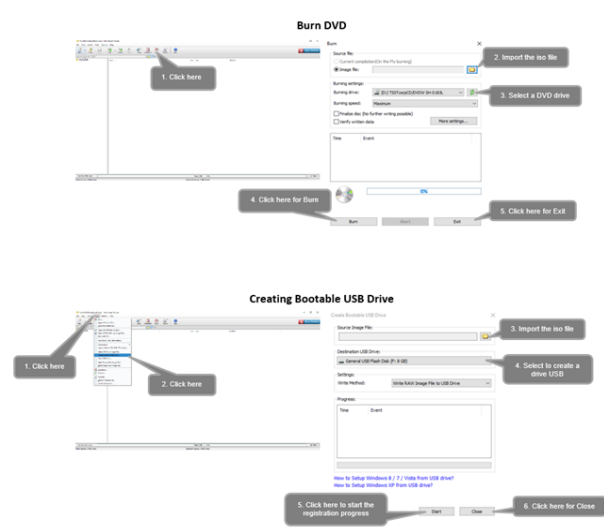

Fig. 1. Burning Ubuntu's ISO image to DVD or USB

\section{Install Ubuntu -}

Ubuntu's installation is shown in the figures below:

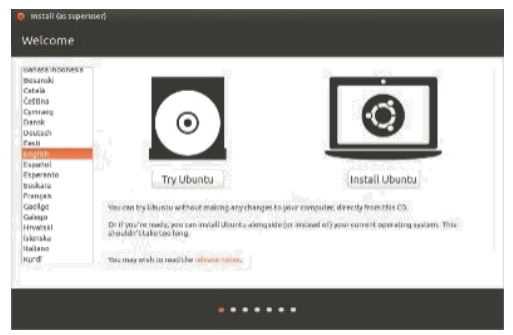

Fig. 2.Ubuntu installation \#1: Ubuntu's language selection

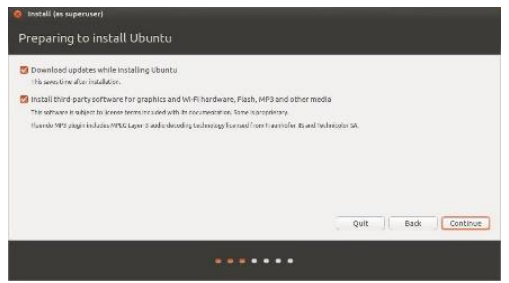

Fig. 3.Ubuntu installation \#2: Select updates' and third party software's installation 
International Journal of Engineering Applied Sciences and Technology, 2019

Vol. 4, Issue 5, ISSN No. 2455-2143, Pages 444-454

Published Online September 2019 in IJEAST (http://www.ijeast.com)

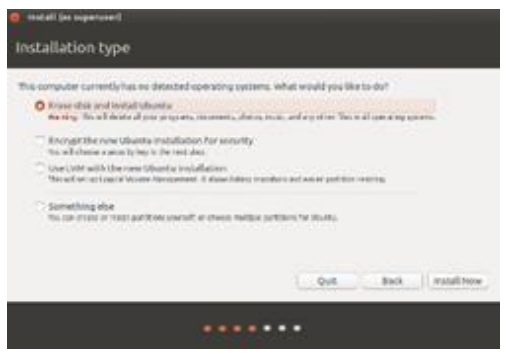

Fig. 4. Ubuntu installation \#3: Select installation type

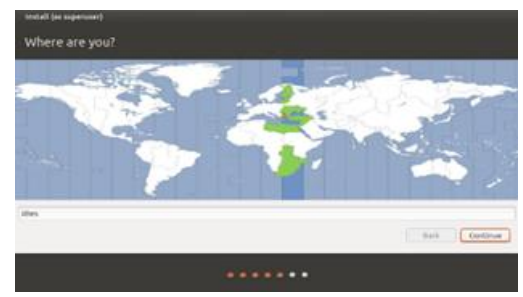

Fig. 5.Ubuntu installation \#4: Select location

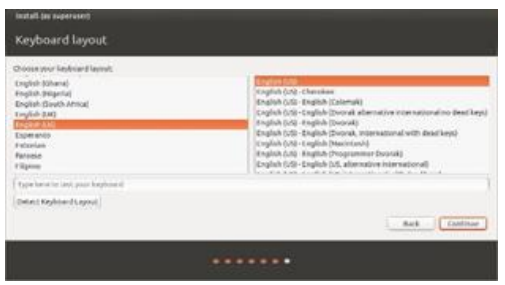

Fig. 6. Ubuntu installation \#5: Select keyboard layout

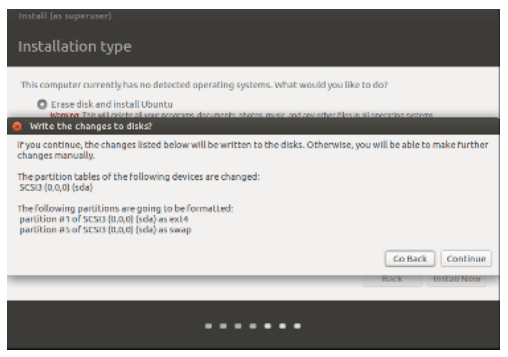

Fig. 7.Ubuntu installation \#5: Select keyboard layout

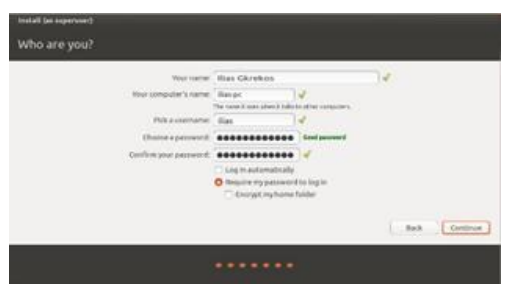

Fig. 8.Ubuntu installation \#6: Setup user account
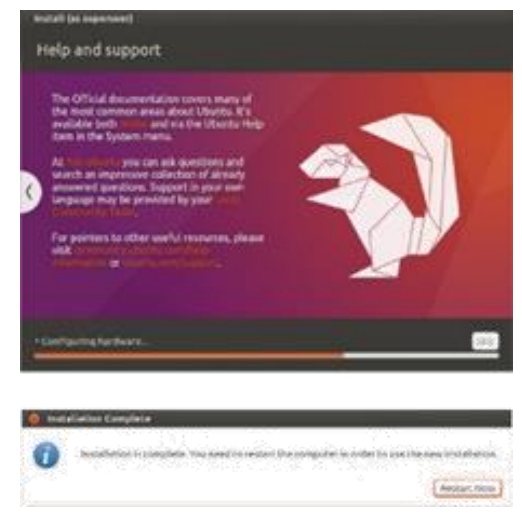

Fig. 9.Ubuntu installation \#8: Installation procedure

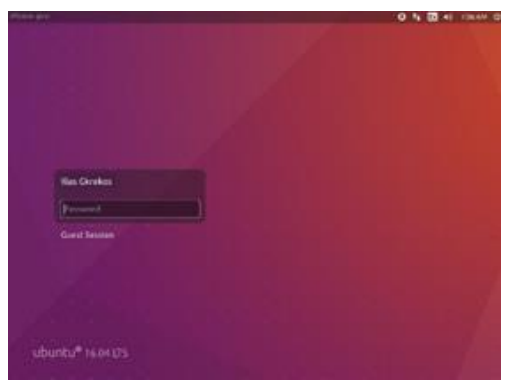

Fig. 10. Ubuntu installation \#9: Login screen - Installation finished

\section{The Ubuntu Desktop}

Ubuntu Desktop uses Unity as the default desktop environment. The Ubuntu desktop consists of the desktop background, a vertical bar called the Launcher and a horizontal bar called the menu bar [16].

Figure 11 shows Dash. Dash is the icon at the top of the Launcher. It's a tool that helps us find files, folders, and applications on our computer quickly. We open the Dash from the Launcher or by pressing the Super button. After clicking the Dash icon our desktop will display a translucent window with a search bar on top. Dash contains six categories listed as lenses by default and serving as specialized search categories at the bottom of the Dash. The six lenses installed by default are: Home lens $(\boldsymbol{\boldsymbol { \imath }})$, Applications lens ( $(\boldsymbol{\wedge})$, Files and Folders lens

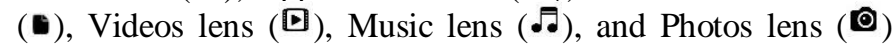
[16].

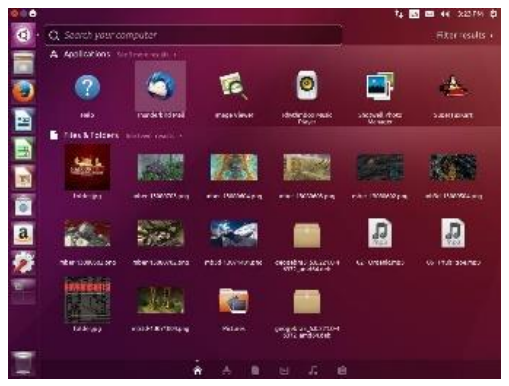


Fig. 11. Dash: Ubuntu's tool for files, folders \& applications

Figure 12 shows Unity's keyboard shortcuts. By holding and holding the Super key, a list of the most useful keyboard shortcuts will appear on our desktop [16].

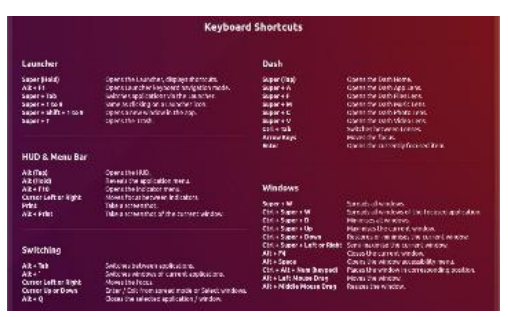

Fig. 12. Ubuntu Unity's keyboard shortcuts

Ubuntu uses Files file manager by default to browse our personal files and personal directories. The Files file manager is the same as Windows Explorer using Microsoft Windows and the Finder using OS X [16].

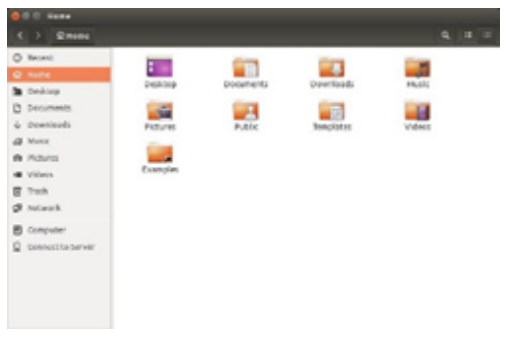

Fig. 13. Ubuntu's Files (file manager application)

To change your desktop customizations right-click on the background and choose Change Desktop Background or go on System Settings - Appearance. Select the Look tab (Figure 14).

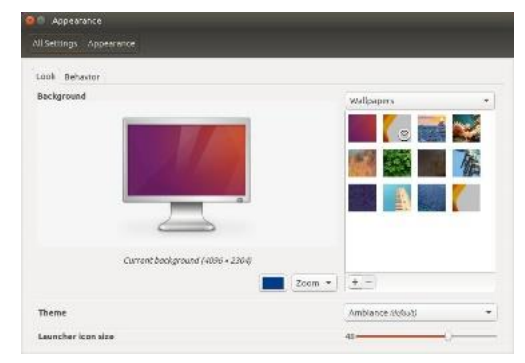

Fig. 14. Ubuntu's system settings

Ubuntu has built-in tools designed to make it easier for people with disabilities to use the computer. We can find these tools and adjust them according to our needs go on System Settings (picture 3.5) • Universal Access (Figure 15) [16].

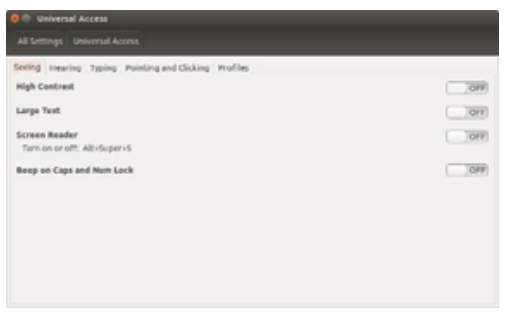

Fig. 15. Ubuntu's universal access

\section{UBUNTU'S APPLICATIONS AND PROGRAMS}

The applications that are underlined are pre-installed, while other applications must be downloaded and be installed through Ubuntu's Software Center. Applications with an asterix can be downloaded from their official web site. The programs that use the Ubuntu are the following [17]:

- Office Suite: LibreOffice, KOffice.

- Email Applications: Mozilla Thunderbird, KMail.

- Web Browsers: Mozilla Firefox, Google Chrome*, Opera*, Epiphany, Midori, Chromium.

- PDF Readers: Evince, Okular.

- Multimedia Players: Totem, VLC, Banshee, SMPlayer, Parole Media Player, Tomahawk, Internet DJ Console, KMPlayer.

- Music Players and Podcatchers: Rhythmbox, VLC, Audacity.

- CD/DVD Burning: Brasero, K3b, SimpleBurn, Xfburn.

- Photo Management: Shotwell, gThumb, Gwenview.

- Graphics Editors: GIMP, Inkscape.

- Instant Messaging: Empathy, Pidgin, Kopete.

- VoIP Applications: Skype, Ekiga.

- $\quad$ BitTorrent Clients: Transmission, Deluge, Vuze.

\section{UBUNTU'S NETWORK CONNECTIONS}

Ubuntu can connect to the Internet using a wired, wireless, or dialup connection. Ubuntu also supports mores advanced connection methods DSL networks and VPN connection. Through Network manager (Figure 16) located on the menu bar is the processing and management of the connections we mentioned [18]. 


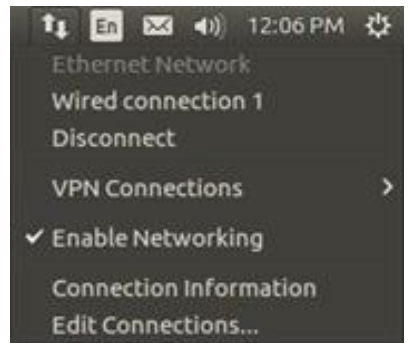

Fig. 16. Ubuntu's Files (file manager application)

\section{HARDWARE}

A wide range of hardware is supported in the Ubuntu operating system. In order to recognize the hardware available on our computer, download and install the Sysinfo (Figure 17) application through the Ubuntu Software Center. This program provides us with information about the devices (hardware) of our system.

The hardware driver is basically a piece of code located within a file and explains the computer how you need to use the hardware. Every item that the computer contains (e.g. graphics card, printer, CD/DVD, hard drives, etc.) requires a driver to work. To manage the settings of each hardware we go System Settings (Figure 14) and we choose the hardware we want in the hardware section (Figure 18) [19].

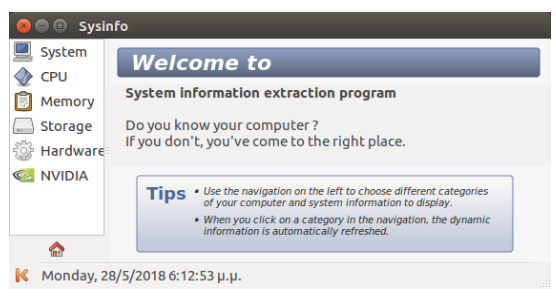

Fig. 17. Sysinfo application

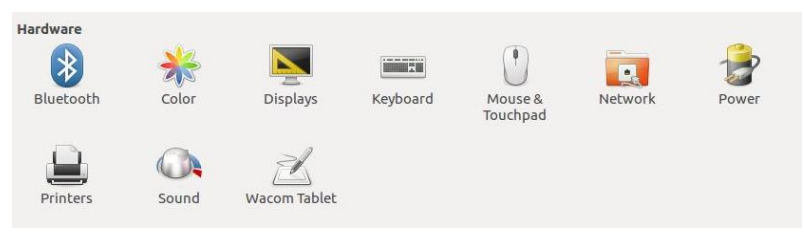

Fig. 18. System settings - Hardware section

\section{SOFTWARE}

\section{A. Package management system -}

Package Management is a collection of tools through which our software can be installed, upgraded, deleted and configured. A packet management system contains a software database called a repository where the individual software is stored in a collection called a package. Packages in a nutshell contain software in a ready-to-install format and have the .deb extension in Ubuntu. Ubuntu uses a package management system called Advanced Packaging Tool or short apt. By default, Ubuntu provides a centralized point with two different ways to browse the repositories for searching, installing, and removing software and are as follows [20]:

- The Ubuntu Software application

- Command line apt-get

\section{B. Ubuntu Software Center -}

In Ubuntu, the fastest and easiest way to find and install new applications or remove applications is the Software Center (Figure 19).

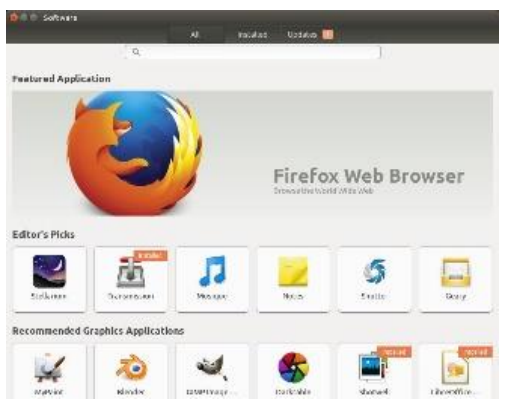

Fig. 19. Ubuntu's Software Center

Through the Ubuntu Software Center, we have access to a variety of applications that are available for download or are commercially available. Every application in the Software Center contains ratings and reviews. The applications used by the Ubuntu Software Center are available in the official Ubuntu repositories. To start Ubuntu Software, click on its icon in the Launcher or click on the Dash and search for Ubuntu Software [20].

\section{Managing additional software -}

\section{Software Sources}

The applications provided by the Ubuntu Software Center are only applications that are located in repositories that are enabled. To add or remove repositories we go to System Settings and click on Software \& Updates and then select the tab Ubuntu Software (Figure 20) [20].

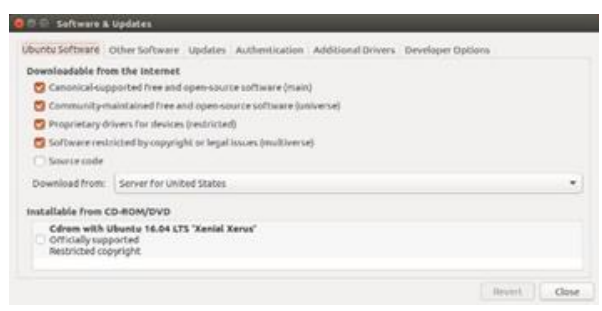

Fig. 20. Ubuntu's Files (file manager application)

\section{Adding more software repositories}

A PPA is a personal packet file. A PPA is a storage file that contains custom software that includes packages that are either 


\section{International Journal of Engineering Applied Sciences and Technology, 2019 \\ Vol. 4, Issue 5, ISSN No. 2455-2143, Pages 444-454 \\ Published Online September 2019 in IJEAST (http://www.ijeast.com)}

not in Ubuntu's official repositories or packs new packages of packages in Ubuntu official repositories. To find the PPA of an application that is not installed in Ubuntu's official repositories, we can use the Launchpad site https://launchpad.net/ that contains all PPAS. When we find the PPA of the application that we want to install, we copy PPA which is of form ppa: test-ppa/example. Once we have copied the PPA, we go to the Software and Updates window and select the Other Software tab to add this PPA [20].

\section{Manual software installation -}

If there are software packages that are not contained in a repository, installation of these packages should be done manually. Ubuntu packages have the .deb extension. Such a package is Google Chrome. Clicking on the package will open a page in the Ubuntu Software Center to install this package. We choose Install and start the package installation process from the Ubuntu Software Center exactly the same as installing the packages contained in the Ubuntu Software Center [20].

\section{E. Ubuntu updates -}

Updates to Ubuntu are done through application Software Updater (Figure 21) [20].

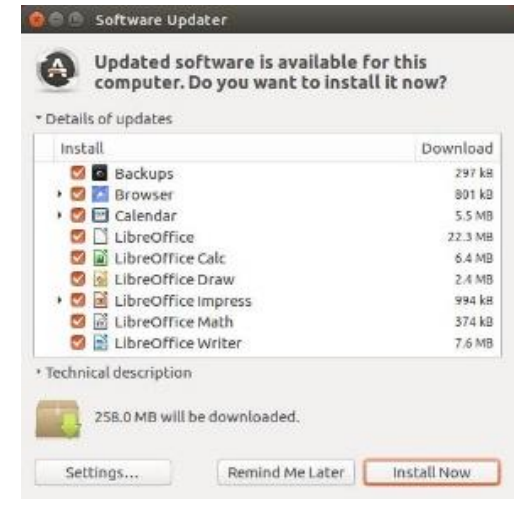

Fig. 21. Ubuntu's Software Updater

\section{THE AdVANCED UBUNTU FUNCTIONS}

\section{A. Terminal}

Through the terminal we have access to the shell. When we open the terminal window and type a command, the shell reads this command and shows us the result. The default shell used by Ubuntu is Bash. To open the terminal, go to Dash and type a terminal and click the Terminal application or use the Ctrl + $A l t+T$ key combination.

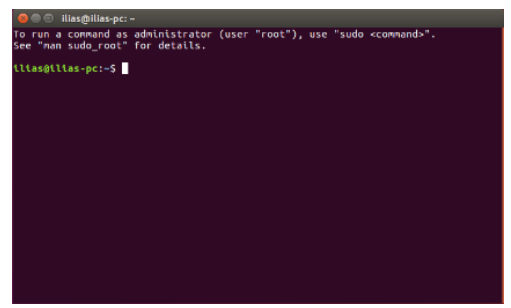

Fig. 22. Ubuntu's Bash (teminal)

By opening the terminal window (Figure 22) at the top left of the window, we see the following:

ilias: login name,

ilias-pc: computer name,

$\sim:$ means it is the current directory of the current user,

$\$$ : The dollar means we're a regular user and not a root.

The blinking block is the cursor where we type our commands. Any command we type in the terminal except that it can be used as a whole can be followed by some parameters. Parameters are special options that are placed at the end of a command so that the command changes the way it is interpreted. For example, using the -h or - -help parameter at the end of a command, we have a short description of the command and what parameters this command can use. Some of the basic commands for the command line Bash are the following [21]:

ls: command used to display the list of files in the current directory.

cd: command used to change a directory.

mv: command used to move or rename files or folders.

man: command used to display a help manual for Linux commands.

mkdir: command used to create folders.

rmdir: command used to remove folders.

rm: command used to remove files.

touch: command used to create blank files

clear: command used to clean the terminal window.

locate: command that is used to search for files by typing their name.

pwd: command used to display the current directory.

To see commands A-Z for a command line Bash you visit the web page https://ss64.com/bash/ [22].

\section{B. File System Ubuntu}

Figure 23 lists some of the major directories contained in the root directory of the Ubuntu file system [23].

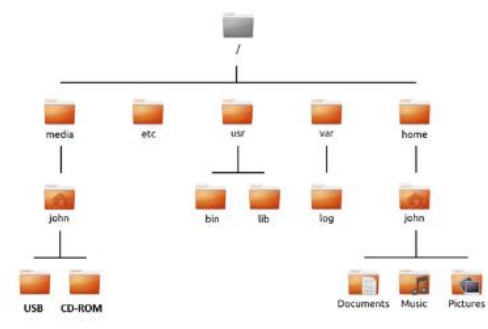


Fig. 23. Ubuntu's file system

/: root directory containing all other directories and files in our system.

/bin and /sbin: Many essential system applications (equivalent to $\mathrm{C}$ : $\backslash$ Windows).

/etc: System-wide configuration files.

/home: Each user will have a subdirectory to store personal files (for example, /home/yourusername) which is equivalent to C:IUsers or C: Documents and Settings in Microsoft Windows.

/lib: Library files, similar to .dll files on Windows.

/media: Removable media (cd-roms and usb drives) will be mounted in this directory.

/root: This contains the root user's files (not to be confused with the root directory).

/usr: Pronounced "user," it contains most program files (not to be con-fused with each user's home directory). This is equivalent to C:IProgram Files in Microsoft Windows.

/var/log: Contains log files written by many applications.

\section{B. Running Windows Programs on Ubuntu}

As mentioned above, many of the programs we use in Microsoft Windows in Ubuntu can't be used because they are not supported for this operating system (e.g. Microsoft Office). In order to overcome this problem and to be able to use Ubuntu, Windows developers have created Wine (Wine Is Not an Emulator) [24].

What is Wine?

Wine is an application through which users of the GNU/Linux and OS X operating systems can install and run Microsoft Windows programs on their system. Several Windows programs are compatible with Wine when running on the GNU/Linux and OS X operating systems. Programs that may not be compatible with Wine need to install additional components such as the Microsoft NET Framework (e.g. Microsoft Office) [24].

\section{Installing Wine}

Step1: Open the terminal and type: sudo apt-add-repository ppa:ubuntu- wine/ppa. This will install the Official Wine PPA.

Step2: After the terminal has finished installing the Wine PPA, type: sudo apt update. This will update the PPA list [24].

Step3: Once the terminal has finished refreshing the PPA list, type: sudo apt install -y wine1.8 winetricks. This will install Wine and Winetricks. Winetricks is a software center for Wine, and is, in most cases, optional [24].

During the installation of Wine and Winetricks, you will have to accept the Microsoft End User License Agreement and the Microsoft Core Fonts License Agreement so that the Microsoft fonts and native files can be installed (Figure 24) [24].

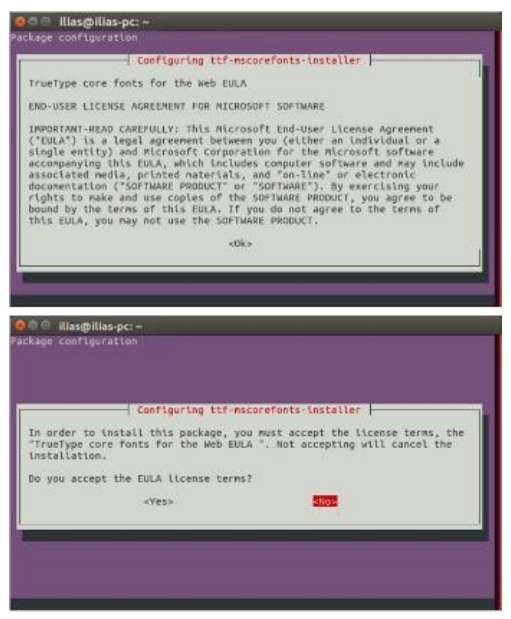

Fig. 24. Wine's installation

\section{Configuring Wine}

For changing Wine settings such as the Microsoft Windows theme or the version of Windows that we want to use open the Dash and search for Configure Wine (Figure 25) [24]

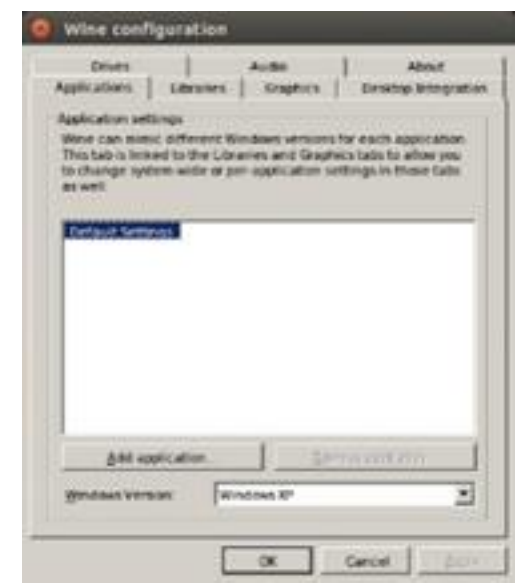

Fig. 25. Ubuntu's Files (file manager application)

\section{Wine \& Microsoft.NET Framework}

The Microsoft .NET Framework has been created by Microsoft and is a program that is required to run applications. Microsoft.NET Framework. To Wine supports several versions of the Microsoft .NET Framework in order to run the newer Microsoft applications but also other partners. To see which versions of Microsoft .NET Framework are compatible with Wine, we go to webpage: https://appdb.winehq.org/objectManager.php?sClass=applicati on\&iId=2586 [24]

\section{PROBLEMS AND SOLUTIONS IN UBUNTU}

\section{A. Ubuntu fails to start after I've installed Windows}




\section{International Journal of Engineering Applied Sciences and Technology, 2019 \\ Vol. 4, Issue 5, ISSN No. 2455-2143, Pages 444-454 \\ Published Online September 2019 in IJEAST (http://www.ijeast.com)}

We start by placing the boot drive we used to install it on our computer and restarting and starting from the boot drive instead of the hard drive. Then we just look at the Ubuntu installer's welcome screen to select the language we want and click on the Try Ubuntu button. When Ubuntu starts opening our terminal and typing \$ sudo fdisk -1 and pressing ENTER.

In the terminal we will see the devices in which each operating system is installed in our system (Figure 26).

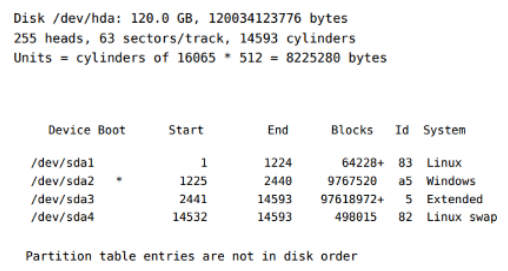

Fig. 26. Terminal: list Ubuntu's devices

The Linux operating system (Ubuntu) is installed on the /dev /sda1 device and the Windows operating system on the /dev/sda2 device. The asterisk on /dev/sda2 indicates that our computer is booting from this device that is Windows (Figure 26). To change this and set our computer to boot from Linux, we will need to connect the existing Ubuntu installation to the temporary troubleshooting conferences.

We type in our terminal:

$\$$ sudo mkdir/ mnt/root.

Then connect the installation of Ubuntu and the new folder by typing on the terminal:

\section{\$ sudo mount /dev/sda1 mnt/root.}

Then type in our terminal: $\$ 1 \mathrm{~s}$ mt/root and this should appear on our terminal (Figure 27).

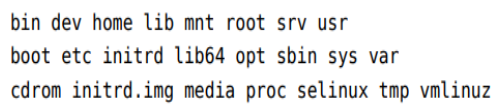

Fig. 27. Terminal listing

Now we are reinstalling GRUB by typing in our terminal:

$\$$ sudo grub-install - -root-directory $=/ \mathrm{mnt} / \mathrm{root} / \mathrm{dev} / \mathrm{sda}$ and this should appear on our terminal (picture 9.3).

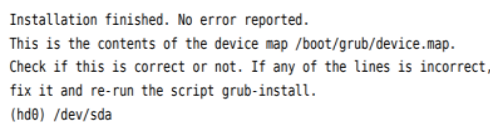

Fig. 28. Terminal listing

Then we will have to disconnect our hard drive to ensure that the drive will not be corrupted when restarting. We type in our terminal:

\$ sudo umount mnt/root.
Finally, we remove the boot drive and reboot our computer and we are ready to use Ubuntu again [25].

\section{B. Reset Password}

If we forget about our password, we will need to restore it using the recovery mode. We first turn off our computer and reboot it. We select from GRUB Advanced options for Ubuntu (Figure 25) and then choose recovery mode.

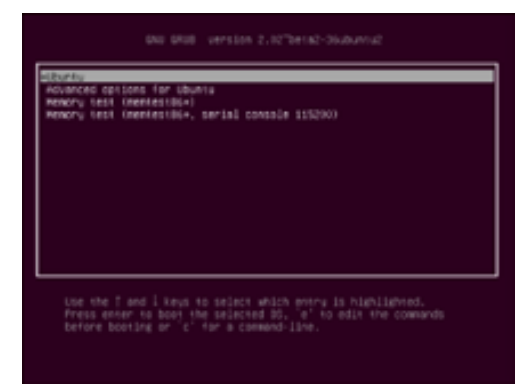

Fig. 29. GRUB advanced options

We wait for Ubuntu to start and when this happens on our screen, the recovery mode menu will appear. We select root and then press ENTER. Now our screen at the bottom will display the function of the terminal which we handle as root and will be in the form:

root@ubuntu: \#

where ubuntu the name of our computer we gave.

In this case for example it will be as follows:

root@ilias-pc: \#.

To reset the password we type in the terminal:

\# passwd username.

We replace the username with our username and then Ubuntu will ask us to type a new password. Enter your desired password by pressing ENTER and then type your password again to confirm and press ENTER. Finally, after we reset our password, we return to the normal system environment by typing in the terminal:

\#init 2

Now we can connect to Ubuntu again [26].

\section{UBUNTU AND DERIVATIVES}

\section{A. Alternative interfaces}

Ubuntu, uses Unity to work as a default desktop environment. However, some users prefer to use Ubuntu with a different interface, so Ubuntu distribution distributions have been created with different desktop environments. These distributions are as follows:

Kubuntu: uses KDE for a default desktop environment. 


\section{International Journal of Engineering Applied Sciences and Technology, 2019 \\ Vol. 4, Issue 5, ISSN No. 2455-2143, Pages 444-454 \\ Published Online September 2019 in IJEAST (http://www.ijeast.com)}

Lubuntu: uses LXDE for the default desktop environment.

Xubuntu: uses XFCE for the default desktop environment.

Ubuntu Budgie: uses Budgie for the default desktop environment.

Ubuntu Mate: uses MATE for the default desktop environment.

NOTE: Any distribution of the above mentioned is likely to use different default applications than the default applications we mentioned for Ubuntu [27].

\section{B. Task specific distributions}

\section{Ubuntu Server}

Ubuntu Server is the version of the Ubuntu operating system for servers [28].

\section{Edubuntu}

Edubuntu is a distribution of Ubuntu production that is designed to be used in schools and other educational institutions [28].

\section{Ubuntu Studio}

Ubuntu Studio is specifically designed for users who use computers to create and edit media projects [28].

\section{Mythbuntu}

Mythbuntu is designed to allow users to swipe their computer into an entertainment system [28].

\section{Ubuntu Kylin}

The official chinese version of Ubuntu's operating system is Ubuntu Kylin.

\section{REFERENCE}

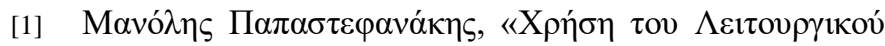

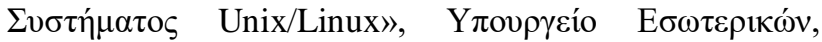

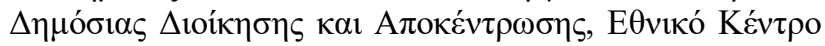

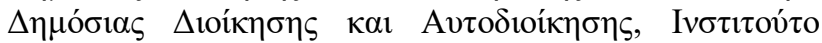

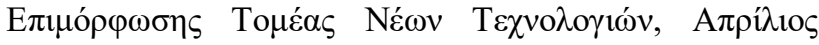
2008, page 13. http://dsphinx.plug.gr/linux_el/unixlinux_usage-ver-1-18.pdf

[2] The Linux Information Project (2005). Kernel DefinitionCategories of Kernels. Available in $\sigma \tau \mathrm{o}$ http://www.linfo.org/kernel.html.

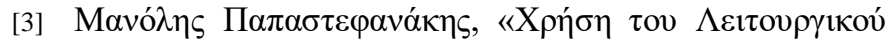

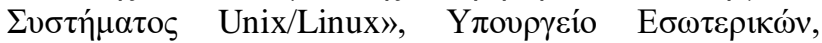

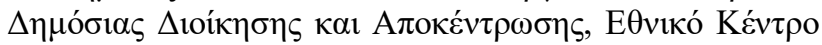

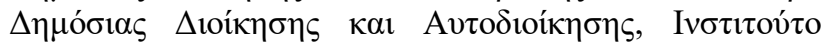

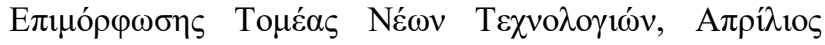
2008, page 14. http://dsphinx.plug.gr/linux_el/unixlinux_usage-ver-1-18.pdf

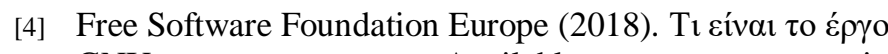
Available in https://fsfe.org/freesoftware/basics/gnuproject.el.html.

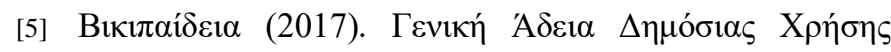
GNU. Available in https://el.wikipedia.org/wiki/\%CE\%93\%CE\%B5\%CE\%B D\%CE\%B9\%CE\%BA\%CE\%AE_\%CE\%86\%CE\%B4\% CE\%В5\%СЕ\%В9\%СЕ\%В1_\%СE\%94\%СЕ\%В7\%СЕ\% ВC\%СF\%8С\%СF\%83\%СЕ\%В9\%СE\%B1\%CF\%82_\%C E\% $\% 7 \% \mathrm{CF} \% 81 \% \mathrm{CE} \% \mathrm{AE} \% \mathrm{CF} \% 83 \% \mathrm{CE} \% \mathrm{~B} 7 \% \mathrm{CF} \% 82$ GNU.

[6] Ankush Das (2017). 11 Reasons Why Linux Is Better Than Windows. Available in https://itsfoss.com/linuxbetter-than-windows/.

[7] Charles E. Craig (2016). Advantages and Disadvantages of Linux. Available in https://renewablepcs.wordpress.com/aboutlinux/advantages-of-using-linux/.

[8] Matctelt Garrels, «Bash Guide for Beginners», 2008, page $6 . \quad$ https://www.tldp.org/LDP/Bash-BeginnersGuide/Bash-Beginners-Guide.pdf

[9] The Ubuntu Manual Team, «Getting Started with Ubuntu 16.04», 2016, page 97-98. http://files.ubuntumanual.org/manuals/getting-started-withubuntu/16.04/en_US/screen/Getting\%20Started\%20with $\% 20$ Ubuntu\%2016.04.pdf

[10] Jason Cannon, «Linux Succinctly», Syncfusion Inc., Morrisville USA, 2014, page 10. https://universalflowuniversity.com/Books/Computer\%20 Programming/Linux\%20Programming/Linux\%20Succinc tly\%20by\%20Jason\%20Cannon.pdf

[11] James Kent Lewis, «Linux Utilities Cookbook», Packt Publishing Ltd, Birmingham UK, 2013, page 21. https://ia802301.us.archive.org/30/items/LewisJamesLinu xUtilitiesCookbook/Lewis\%2C\%20James\%20\%20Linux\%20Utilities\%20Cookbook.pdf

[12] Jason Cannon, «Linux Succinctly», Syncfusion Inc., Morrisville USA, 2014, page 11-12. https://universalflowuniversity.com/Books/Computer\%20 Programming/Linux\%20Programming/Linux\%20Succinc tly\%20by\%20Jason\%20Cannon.pdf

[13] Wikipedia (2018).Ubuntu (operating system). Available in https://en.wikipedia.org/wiki/Ubuntu_(operating_system)

[14] The Ubuntu Manual Team, «Getting Started with Ubuntu 16.04», 2016, page 9. http://files.ubuntumanual.org/manuals/getting-started-withubuntu/16.04/en_US/screen/Getting\%20Started\%20with $\% 20$ Ubuntu\%2016.04.pdf

[15] The Ubuntu Manual Team, «Getting Started with Ubuntu 16.04», 2016, page 11-17. http://files.ubuntumanual.org/manuals/getting-started-with- 
ubuntu/16.04/en_US/screen/Getting\%20Started\%20with \%20Ubuntu\%2016.04.pdf

[16] The Ubuntu Manual Team, «Getting Started with Ubuntu 16.04», 2016, page 19-32. http://files.ubuntumanual.org/manuals/getting-started-withubuntu/16.04/en_US/screen/Getting\%20Started\%20with $\% 20$ Ubuntu\%2016.04.pdf

[17] The Ubuntu Manual Team, «Getting Started with Ubuntu 16.04», 2016, page 37-38. http://files.ubuntumanual.org/manuals/getting-started-withubuntu/16.04/en_US/screen/Getting\%20Started\%20with $\% 20$ Ubuntu\%2016.04.pdf

[18] The Ubuntu Manual Team, «Getting Started with Ubuntu 16.04», 2016, page 39. http://files.ubuntumanual.org/manuals/getting-started-withubuntu/16.04/en_US/screen/Getting\%20Started\%20with \%20Ubuntu\%2016.04.pdf

[19] The Ubuntu Manual Team, «Getting Started with Ubuntu 16.04», 2016, page 79. http://files.ubuntumanual.org/manuals/getting-started-withubuntu/16.04/en_US/screen/Getting\%20Started\%20with \%20Ubuntu\%2016.04.pdf

[20] The Ubuntu Manual Team, «Getting Started with Ubuntu 16.04», 2016, page 87-95. http://files.ubuntumanual.org/manuals/getting-started-withubuntu/16.04/en_US/screen/Getting\%20Started\%20with \%20Ubuntu\%2016.04.pdf

[21] The Ubuntu Manual Team, «Getting Started with Ubuntu 16.04», 2016, page 98. http://files.ubuntumanual.org/manuals/getting-started-withubuntu/16.04/en_US/screen/Getting\%20Started\%20with \%20Ubuntu\%2016.04.pdf

[22] SS64 (2018). An A-Z Index of the Bash command line for Linux.Available in https://ss64.com/bash/.

[23] The Ubuntu Manual Team, «Getting Started with Ubuntu 16.04», 2016, page 99. http://files.ubuntumanual.org/manuals/getting-started-withubuntu/16.04/en_US/screen/Getting\%20Started\%20with $\% 20$ Ubuntu\%2016.04.pdf

[24] The Ubuntu Manual Team, «Getting Started with Ubuntu 16.04», 2016, page 106-109. http://files.ubuntumanual.org/manuals/getting-started-withubuntu/16.04/en_US/screen/Getting\%20Started\%20with \%20Ubuntu\%2016.04.pdf

[25] The Ubuntu Manual Team, «Getting Started with Ubuntu 16.04», 2016, page 111-112. http://files.ubuntumanual.org/manuals/getting-started-withubuntu/16.04/en_US/screen/Getting\%20Started\%20with \%20Ubuntu\%2016.04.pdf

[26] The Ubuntu Manual Team, «Getting Started with Ubuntu 16.04», 2016, page 112-113. http://files.ubuntumanual.org/manuals/getting-started-with-
ubuntu/16.04/en_US/screen/Getting\%20Started\%20with \%20Ubuntu\%2016.04.pdf

[27] The Ubuntu Manual Team, «Getting Started with Ubuntu 16.04», 2016, page 118. http://files.ubuntumanual.org/manuals/getting-started-withubuntu/16.04/en_US/screen/Getting\%20Started\%20with $\% 20$ Ubuntu\%2016.04.pdf

[28] The Ubuntu Manual Team, «Getting Started with Ubuntu 16.04», 2016, page 119. http://files.ubuntumanual.org/manuals/getting-started-withubuntu/16.04/en_US/screen/Getting\%20Started\%20with $\% 20$ Ubuntu\%2016.04.pdf 